\title{
Individualized lung protective ventilation vs. conventional ventilation during general anesthesia in laparoscopic total hysterectomy
}

\author{
JING LIU $^{1 *}$, XINHUA HUANG $^{1 *}$, SIPING HU ${ }^{2}$, ZHIPENG MENG ${ }^{2}$ and HUANZHONG HE ${ }^{2}$ \\ ${ }^{1}$ Department of Anesthesiology, Huzhou Maternity and Child Healthcare Hospital; ${ }^{2}$ Department of Anesthesiology, \\ Huzhou Central Hospital, Affiliated Central Hospital of Huzhou University, Huzhou, Zhejiang 313000, P.R. China
}

Received June 8, 2019; Accepted November 21, 2019

DOI: $10.3892 /$ etm.2020.8549

\begin{abstract}
Laparoscopic total hysterectomy is performed by carbon dioxide insufflation, Trendelenburg position and mechanical ventilation of patients under general anesthesia. However, this may induce pulmonary atelectasis and/or hyperdistention of the lungs. Multiple studies have indicated that mechanical ventilation with the use of low tidal volumes, moderate positive end-expiratory pressure (PEEP) and regular alveolar recruitment maneuvers may improve post-operative outcomes. However, the benefits of an individualized level of PEEP have not been clearly established. In the present study, it was hypothesized that a moderate fixed PEEP may not suit all patients and an individually-titrated PEEP during anesthesia may improve the peri-operative pulmonary oxygenation function. The aim of the present study was to compare the pulmonary oxygenation function and post-operative pulmonary complications (PPCs) in patients receiving individualized lung-protective mechanical ventilation (LPV) vs. conventional ventilation $(\mathrm{CV})$ during laparoscopic total hysterectomy. The present study was a randomized double-blinded clinical trial on 87 patients who were randomly divided to receive $\mathrm{CV}$ or protective ventilation (PV). An optimal individualized PEEP value was determined using a static pulmonary compliance-directed PEEP titration procedure. Pulmonary oxygenation function, serum inflammatory factors, including interleukin-8 and Clara cell protein 16, the incidence of PPCs and the post-operative length of stay were also determined. Patients in the PV group
\end{abstract}

Correspondence to: Dr Zhipeng Meng or Dr Huanzhong He, Department of Anesthesiology, Huzhou Central Hospital, Affiliated Central Hospital of Huzhou University, 198 Redflag Road, Wuxing Area, Huzhou, Zhejiang 313000, P.R. China

E-mail: 21866063@qq.com

E-mail: 849796799@qq.com

Key words: lung-protective mechanical ventilation, individualized positive end-expiratory pressure, laparoscopic total hysterectomy, pulmonary oxygenation function, post-operative pulmonary complications exhibited improved pulmonary oxygenation function during and after the operation. The total percentage of PPCs during the first 7 days after surgery was significantly lower in the PV group compared with those in the CV group. In conclusion, as compared to $\mathrm{CV}$, intra-operative individualized LPV significantly improved pulmonary oxygenation function and reduced the incidence of PPCs during the first 7 days after laparoscopic total hysterectomy (Clinical trial registration no. ChiCTR1900027738).

\section{Introduction}

Laparoscopic total hysterectomy is a major abdominal surgery with an operating time of several hours. It is performed using $\mathrm{CO}_{2}$ insufflation and mechanical ventilation on patients in the Trendelenburg position under general anesthesia. Inappropriate mechanical ventilation settings during general anesthesia may aggravate and even initiate lung damage in patients with normal lungs, which may theoretically lead to ventilator-induced lung injury (VILI) $(1,2)$. The expression of interleukin-8 (IL-8) is stimulated by the classic pro-inflammatory cytokines tumor necrosis factor and IL-1, which are released early in the inflammatory response. In particular, IL-8 is known to participate in the development of lung injury in adults with acute respiratory distress syndrome (ARDS) (3-5). Clara cell protein $16(\mathrm{CC} 16)$ is a lung secretory protein (6) with anti-oxidant and anti-inflammatory properties that is regarded as a sensitive biomarker to potentially identify lung injury in surgical patients within hours $(7,8)$. Multiple review articles suggested that lung-protective mechanical ventilation (LPV) is beneficial during anesthesia in patients with healthy lungs $(9,10)$, and in individuals with ARDS in intensive care units (ICU) (11). Decades of study have demonstrated that LPV may also reduce VILI (12), via the production of low tidal volumes (TV) (13) and higher positive end-expiratory pressure (PEEP) (14), as well as the performance of regular alveolar recruitment maneuvers (ARM) (15). The effects of different components of ventilation on improving post-operative outcomes in patients with normal lungs following surgery have been broadly explored (16). Relevant studies have concluded that only low TV ventilation reduces post-operative pulmonary complications (PPCs) effectively and the efficacy afforded 
by other components, including the level of PEEP, remains controversial (17-19). Moderate fixed PEEP may not suit each patient and it is critical to determine individualized PEEP to stabilize the lung and maximize lung protection thereby reducing the risk of VILI. Properly adjusted PEEP may have a significant protective effect, whereas inadequate PEEP values may promote pulmonary atelectasis and/or hyperinflation of dependent lung tissue (20-23). Therefore, in order for PEEP to be effective, it must be personalized to suit the individual lung physiology of each patient. However, the efficacy of using individualized PEEP values has not been studied thoroughly in laparoscopic total hysterectomy.

Multiple methods of personalizing PEEP have been investigated, including inflection points on the pressure/volume curve, dead space (VD) to TV ratio (VD/TV) or static lung compliance (Cstat)-directed techniques (24). In the present clinical trial, optimal PEEP values were determined during a Cstat-directed PEEP titration procedure to protect from hyperdistention and regular ARM was performed by volume-controlled ventilation to prevent atelectasis.

The aim of the present study was to evaluate the benefit of intra-operative individualized LPV on pulmonary oxygenation function and the incidence of PPCs in patients scheduled for laparoscopic total hysterectomy surgery. It was hypothesized that intra-operative individualized LPV may improve pulmonary oxygenation function and decrease the total occurrence of PPCs compared to conventional ventilation during anesthesia in patients undergoing laparoscopic total hysterectomy surgery.

\section{Materials and methods}

Study design. All patients provided written informed consent prior to inclusion in the study. The present study was a randomized, double-blinded, controlled, investigator-initiated clinical trial and was approved by the Ethical Committee of Huzhou Maternal and Child Healthcare Hospital (approval no. 20180206-9; Huzhou, China). Investigators randomly assigned participants to the PV group or the CV group at a ratio of 1:1. Concealed randomization was performed using Random Allocation Software (Windows software, version 1.0; Microsoft Corp.). The group-identification information was stored in sealed and numbered envelopes. Participants were included and allocated in numerical order.

The anesthetist was responsible for the collection of patient data during surgery. Ventilator settings recorded during anesthesia were concealed in the case report form. The surgeon responsible for the patient was not informed of the ventilator settings. Physicians that were not involved in the care of the patient during anesthesia and surgery performed post-operative evaluation. Anesthesia recordings and ventilator settings during surgery were concealed from the post-operative physicians and nurses.

Inclusion and exclusion criteria. Patients who were scheduled for elective laparoscopic total hysterectomy surgery under general anesthesia between January 2017 and January 2019 at Huzhou Maternal and Child Healthcare Hospital were included in the present study. Patients were eligible for participation if they conformed to the following criteria: American
Society of Anesthesiologists (ASA) physical status I-III; age of $\geq 18$ years; body mass index (BMI) $<30 \mathrm{~kg} / \mathrm{m}^{2}$; candidates for elective laparoscopic total hysterectomy surgery under general anesthesia with an expected duration of $>2 \mathrm{~h}$. The exclusion criteria were as follows: Individuals who refused or were unable to provide informed consent, or were participating in another interventional study; neuropathy or any neuromuscular disease; thoracic deformity and intrathoracic diseases (e.g. mediastinal tumor or chest tumor); severe cardiac disease defined as New York Heart Association grade III or IV, acute coronary syndrome or persistent ventricular tachyarrhythmias; any previous lung surgery or history of pulmonary disease; use of positive pressure ventilation prior to surgery [e.g. continuous positive airway pressure for sleep obstructive apnea syndrome (CPAP)]; liver cirrhosis (Child-Pugh score B or C); chronic renal failure with dialysis; allergy to local anesthetic; emergency surgery; or the requirement for the patient to be transferred to the ICU after surgery.

Standard procedures. All patients accepted a standard procedure of general anesthesia induction comprising intravenous midazolam hydrochloride $(0.05 \mathrm{mg} / \mathrm{kg}$; Jiangsu Enhua Medical Co.), sufentanyl $(0.6 \mu \mathrm{g} / \mathrm{kg}$; Yichang Renfu Medical Co.), propofol (2 mg/kg; Xi An Li Bang Medical Co.) and rocuronium bromide $(0.6 \mathrm{mg} / \mathrm{kg}$; Zhe Jiang Xian Ju Medical Co.). After $3 \mathrm{~min}$, patients were intubated with a tracheal tube (internal diameter, $7 \mathrm{~mm}$ for females), and the tube cuff pressure was adjusted between 20 and $25 \mathrm{cmH}_{2} \mathrm{O}$. Maintenance anesthesia was provided by continuous intravenous propofol infusion $(4-12 \mathrm{mg} / \mathrm{kg} / \mathrm{h})$, remifentanil infusion $(0.05-0.3 \mu \mathrm{g} / \mathrm{kg} / \mathrm{min})$ and sevoflurane inhalation (concentration, 1-3\%) based on the BiSpectral index of the patient, which was maintained at 40-60. As continuous intravenous propofol and remifentanil infusion were provided to maintain anesthesia and inhibit respiration, additional cisatracurium besilate $(5 \mathrm{mg}$ ) was prescribed when clinically indicated to produce further muscle relaxation. Routine intra-operative monitoring was performed continuously using a dedicated monitor, including invasive blood pressure, pulse oximetry, heart rate, end-tidal fractions of carbon dioxide $\left(\mathrm{ETCO}_{2}\right)$ and electrocardiogram. Immediately prior to the end of the operation, tolane setron $(5 \mathrm{mg}$ ) was administered to prevent post-operative nausea and vomiting. After the surgery, patients were directly transported to the post-anesthesia care unit. After tracheal extubation, patients were oxygenated with an inspired oxygen fraction $\left(\mathrm{FiO}_{2}\right)$ of 0.33 through a venturi face mask (Tian Jin Shuang Li Medical Device Co., Ltd).

Following surgery, the patients received intravenous continuous analgesia in the form of sufentanyl $(50 \mu \mathrm{g})$ and dexmedetomidine $(200 \mu \mathrm{g})$, combined with $100 \mathrm{ml}$ normal saline to control pain. The background dose was $2 \mathrm{ml} / \mathrm{h}$ and the bolus dose was $2 \mathrm{ml}$. Post-operative analgesia was provided for at least $48 \mathrm{~h}$ to achieve a visual analogue scale (VAS) score of $<3$ (25). If the VAS score was $\geq 3$, intravenous tramadol (50 mg) was to be administered for remedial analgesia, but none of the patients was given tramadol in the present study as the VAS scores were $\geq 3$. All patients received routine physiotherapy (26) during the post-operative period according to the standard of care at Huzhou Maternal and Child Healthcare Hospital (Huzhou, China). 
Ventilation protocol. In the two groups, the surgery was performed under pneumoperitoneum induced and held using an intra-abdominal pressure of $13-15 \mathrm{mmHg}$ with room-temperature $\mathrm{CO}_{2}$ insufflation. The mechanical ventilation protocol consisted of a volume-controlled mode with an $\mathrm{FiO}_{2}$ of 0.40 and an inspiratory to expiratory ratio of 1:2 in the two groups, which was performed using an S/5 Avance anesthesia machine (Datex Ohmeda; GE Healthcare). The respiratory rate (RR) was regulated to obtain an $\mathrm{ETCO}_{2}$ of $35-45 \mathrm{mmHg}$.

Patients were randomly assigned to the $\mathrm{CV}$ group or the PV group. In the CV group, the ventilation protocol was TV of $9 \mathrm{ml} / \mathrm{kg}$ of ideal body weight (IBW), without PEEP or ARM throughout the surgery. The IBW was calculated according to the following pre-defined formula for females (27): IBW $(\mathrm{kg})=45.5+0.91$ (height in $\mathrm{cm}-152.4$ ).

In the PV group, the ventilation protocol settings were the same as those in the CV group immediately after induction of anesthesia and orotracheal intubation. However, once a steady state had been reached, all patients were submitted to an ARM using sustained airway pressure via the CPAP method, applying TV of $7 \mathrm{ml} / \mathrm{kg} \mathrm{IBW}$ and $30 \mathrm{~cm} \mathrm{H}_{2} \mathrm{O}$ PEEP for $30 \mathrm{sec}$ based on a study by Ruszkai et al (28), followed by a decremental PEEP titration (DPT) procedure directed by Cstat. The depth of anesthesia was regulated to ensure the patients were haemodynamically stable and $\mathrm{PaO}_{2} / \mathrm{FiO}_{2}$ of $\geq 300 \mathrm{mmHg}$ was also guaranteed to assure that the pulmonary oxygenation function was normal. If so, the ARM was considered successful.

The method of DPT was as follows (28): During the PEEP titration procedure, PEEP was decreased from $14 \mathrm{~cm} \mathrm{H}_{2} \mathrm{O}$ by $2 \mathrm{~cm} \mathrm{H}_{2} \mathrm{O}$ every $4 \mathrm{~min}$, until a final PEEP of $6 \mathrm{~cm} \mathrm{H}_{2} \mathrm{O}$ was reached. On each level of PEEP, Cstat was measured by the ventilator. Optimal PEEP was considered as the PEEP value resulting in the highest possible Cstat measurement. After the PEEP titration procedure, an ARM was implemented and lung-protective mechanical ventilation was then performed using the optimal PEEP and TV of $7 \mathrm{ml} / \mathrm{kg}$.

Data source and collection. The following demographic parameters were recorded for the 87 patients enrolled: Age, BMI, ASA physical status and post-operative length of stay (PLOS). The intra-operative data, including operation time, mechanical ventilation time, TV, RR and arousal time (time from the end of the operation to the time the patients woke up) were recorded for the two groups. Breathing mechanics [pulmonary dynamic compliance (Cdyn)] were calculated as TV (peak-PEEP) (24) in the operative period at time-point 1 (T1; after endotracheal intubation), T2 (10 min after pneumoperitoneum), T3 (60 min after pneumoperitoneum) and T4 (10 min after pneumoperitoneum was stopped). Arterial blood gas (ABG) was recorded at T1, T3, T5 (30 min after tracheal extubation) and T6 (the day after the surgery). The lung oxygenation ability was assessed by determining the oxygen index (OI) calculated from the pressure of arterial oxygen $\left(\mathrm{PaO}_{2}\right)$ and $\mathrm{FiO}_{2}$ as $\mathrm{OI}=\mathrm{PaO}_{2} / \mathrm{FiO}_{2}$ (29). Alveolar-arterial differences for oxygen $\left(\mathrm{A}-\mathrm{aO}_{2}\right)$ were calculated from the atmospheric pressure $(\mathrm{PB})$, saturated vapor pressure at room temperature $\left(\mathrm{PH}_{2} \mathrm{O}\right)$, pressure of arterial carbon dioxide $\left(\mathrm{PaCO}_{2}\right)$ and respiration quotient $(\mathrm{R}), \mathrm{PaO}_{2}$ and $\mathrm{FiO}_{2}$ as $\mathrm{A}-\mathrm{aO}_{2}=\left(\mathrm{PB}-\mathrm{PH}_{2} \mathrm{O}\right) \times \mathrm{FiO}_{2}-\mathrm{PaCO}_{2} / \mathrm{R}-\mathrm{PaO}_{2}$ (30), with PB set at $760 \mathrm{mmHg}, \mathrm{PH}_{2} \mathrm{O}$ at $47 \mathrm{mmHg}$ and $\mathrm{R}$ at 0.8 . Saturation of pulse oxygenation $\left(\mathrm{SPO}_{2}\right)$ was recorded on the second day after surgery (D2) and D5. $\mathrm{SPO}_{2}$ was measured in the ward. If the patient was using a nasal oxygen catheter, the catheter was removed for $10 \mathrm{~min}$ and $\mathrm{SPO}_{2}$ was then measured after adaptation on D2 and D5. If $\mathrm{SPO}_{2}$ dropped $<90 \%$ during the adaptive time, the manipulation was stopped and $\mathrm{SPO}_{2}$ was immediately obtained. Venous blood samples were taken at T1, T3, T5 and T6 to assess the systemic inflammatory response. Venous blood samples of the patients were centrifuged at 5,000 x g for $10 \mathrm{~min}$ at $4^{\circ} \mathrm{C}$, and supernatants were separated immediately after collection. All supernatants were stored at $-80^{\circ} \mathrm{C}$ until biochemical measurements were performed. eBioscience Platinum ELISA (Bender MedSystems GmbH) was used to measure the serum levels of IL-8 and CC16. Post-operative follow-up was performed and the total incidence rate of PPCs in the two groups during the first 7 post-operative days was recorded. PPCs were defined as the development of one of seven complications: Atelectasis, pleural effusion, respiratory infection, pneumothorax, bronchospasm, respiratory failure and aspiration pneumonitis during the period of post-operative hospitalization, as reported by Jammer et al (31) and Gallart and Canet (32).

Primary and secondary endpoints. The primary endpoint was the change in pulmonary oxygenation function including OI during the pre-and post-operative period. The secondary endpoints were Cdyn during the operation and the total incidence of PPCs in the first 7 days after surgery. Post-operative pneumonia was diagnosed based on 3 criteria: i) Presence of new pulmonary infiltrates from chest X-ray images; ii) leukocytosis and iii) fever (ear temperature, $\left.\geq 38.0^{\circ} \mathrm{C}\right)(33,34)$.

Statistical analysis. The formula for calculation of the sample size was as follows: $\mathrm{n}=[(\mathrm{Z} \alpha / 2+\mathrm{Z} \beta) 2 \times 2$ (SD) $2 /(\mu 1-\mu 2) 2](35)$, where $\mathrm{n}$ represents the sample size required in each group, $\mu 1$ is the mean of the OI in the PV group, $\mu 2$ is the mean of the OI in the CV group; $\mu 1-\mu 2$ is the clinically significant difference; $\mathrm{Z} \alpha / 2$ represents a $5 \%$ level of significance (1.96); $\mathrm{Z} \beta$ represents $95 \%$ statistical power (1.96) and SD represents the standard deviation, which was 1.195. A pilot study was performed on 16 patients ( 8 in each group) to detect a significant difference in OI between groups, during which $\mu 1$ was measured as 1.05 and $\mu 2$ as 1.25 . The OI was obtained during the pilot study at T3 (60 min after pneumoperitoneum) and T4 (10 min after pneumoperitoneum was stopped). Therefore, $\mathrm{n}$ was equal to 35 for each group, requiring a total sample size of 70 . Considering a $20 \%$ attrition rate, the total sample size was increased to 86 patients (43 patients in each group). Statistical analysis was performed using SPSS version 17 (SPSS Inc.). The normality of distribution of continuous variables was tested using one-sample Kolmogorov-Smirnov test. Continuous data were analyzed using one-way ANOVA to assess the significance of differences in means between and within the groups. If the variance was not homogeneous, a non-parametric test (Kruskal-Wallis H-test for multiple independent samples) was used. Categorical variables were compared using the $\mathrm{RxC} \chi^{2}$ test or Fisher's exact test. In all cases, P-values were two-sided and $\mathrm{P}<0.05$ was considered to indicate a statistically significant difference. 


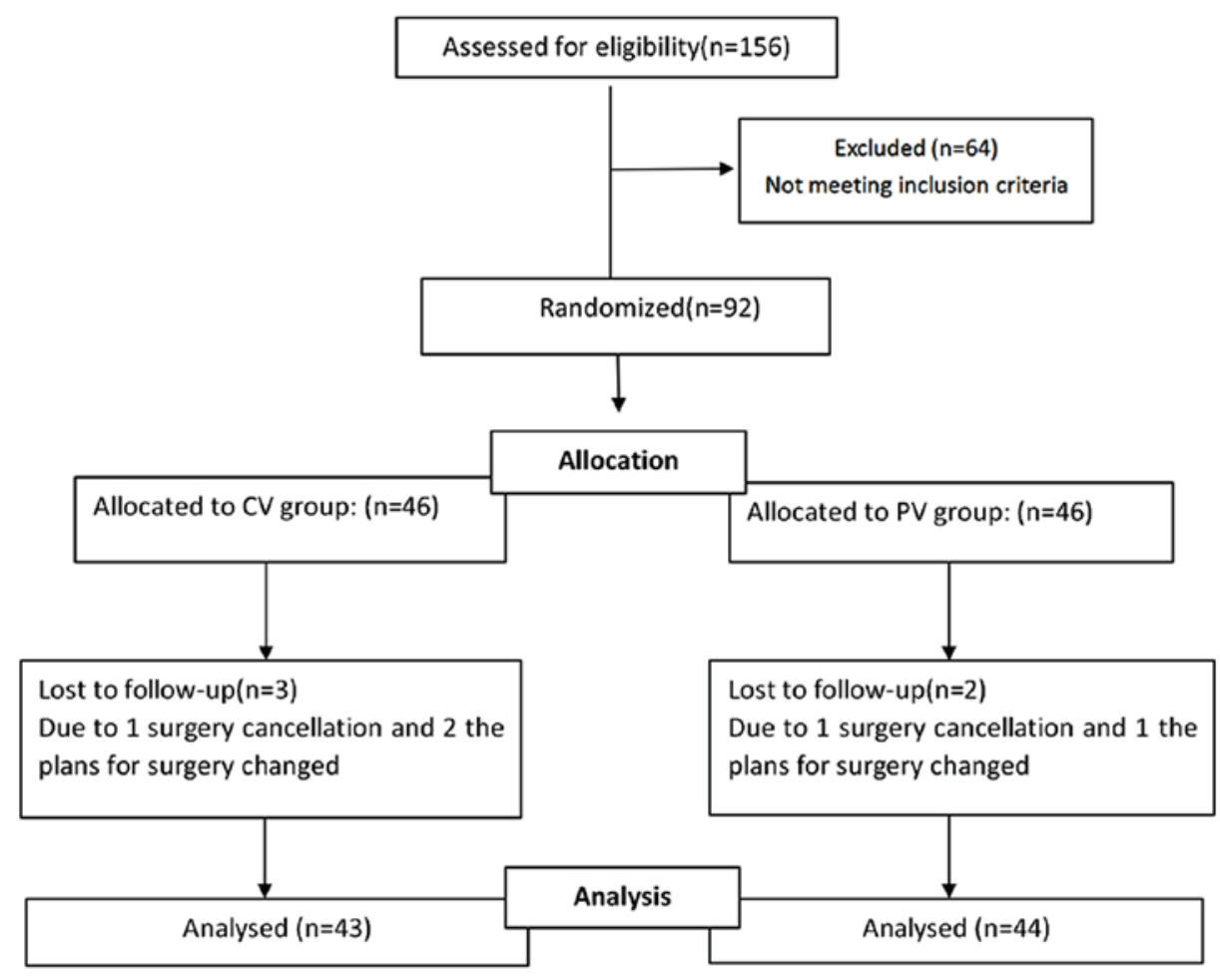

Figure 1. Flow chart depicting the selection of patients for the present study. Patients who were assessed for eligibility and a proportion of them were excluded, as they did not meet the inclusion criteria.

\section{Results}

Patient enrolment and follow-up. A flow chart of the enrolment and follow-up of the patients is provided in Fig. 1. A total of 156 consecutive patients who were scheduled to undergo laparoscopic total hysterectomy surgery were assessed for eligibility. Of these, 64 patients were excluded, as they did not meet the inclusion criteria. The remaining 92 patients were included in the study and were divided into 2 groups with 46 patients each in the CV group and the PV group. The surgery was cancelled for 2 patients assigned to the $\mathrm{CV}$ group and 1 patient assigned to the PV group. In addition, 2 patients assigned to the $\mathrm{CV}$ group and 1 patient assigned to the PV group were excluded, as the plans for surgery changed. Finally, 87 patients were enrolled and included in the final analysis, comprising 43 in the $\mathrm{CV}$ group and 44 in the PV group. In terms of the baseline characteristics, there were no significant differences between the two groups (Table I).

Intra-operative data of the two groups. Compared with those in the CV group, the TV was lower and the RR was higher in the PV group; however, there were no statistically significant differences between the two groups with regard to intra-operative mechanical ventilation time, operation time and arousal time (Table II).

Breathing mechanics during the operative period in the two groups are presented in Fig. 2. In the two groups, Cdyn exhibited a marked decline at $\mathrm{T} 2$ as compared with that at $\mathrm{T} 1$, but no statistical significance was found. Compared with that in the CV group, Cdyn was significantly elevated in the PV group at $\mathrm{T} 3$ and $\mathrm{T} 4(\mathrm{P}<0.05)$.
Pulmonary oxygenation. The pulmonary oxygenation during the operative and post-operative period for the two groups is presented in Figs. 3-5. In the PV group, the OI value was higher and the $\mathrm{A}-\mathrm{aO}_{2}$ was lower compared with those in the $\mathrm{CV}$ group at T3, T5 and T6 $(\mathrm{P}<0.05)$. Furthermore, in the PV group, $\mathrm{SPO}_{2}$ was higher compared with that in the $\mathrm{CV}$ group on $\mathrm{D} 2$ and $\mathrm{D} 5(\mathrm{P}<0.05)$.

Inflammatory factors. The inflammatory factors IL-8 and CC16 in the two groups at T1, T3, T5 and T6 are presented in Figs. 6 and 7. There were no significant differences in IL-8 and $\mathrm{CC} 16$ in the two groups at any time-point $(\mathrm{P}>0.05)$.

Incidence of PPCs. The total incidence of PPCs was compared post-operatively in the two groups during the first 7 days after surgery (Table III). Pneumothorax, bronchospasm, respiratory failure and aspiration pneumonitis did not occur in either group. Compared with the $\mathrm{CV}$ group, there was no difference in the incidence of respiratory infection and pleural effusion in the PV group $(\mathrm{P}>0.05)$. However, the total incidence of PPCs and atelectasis in the $\mathrm{CV}$ group was higher compared with that in the PV group $(\mathrm{P}<0.05)$.

\section{Discussion}

In the present study, pulmonary dynamic Cdyn, pulmonary oxygenation, serum inflammatory factors and the total incidence of PPCs were compared between a CV and a PV group. It was observed that, compared with conventional mechanical ventilation without PEEP, individualized lung PV performed by applying personalized PEEP and regular ARM was associated with improved pulmonary dynamic Cdyn and pulmonary 
Table I. Baseline characteristics of the patients.

\begin{tabular}{lccc}
\hline Parameter & CV group $(\mathrm{n}=43)$ & PV group $(\mathrm{n}=44)$ & P-value \\
\hline Age (years) & $50.32 \pm 9.83$ & $51.08 \pm 8.86$ & 0.72 \\
BMI $\left(\mathrm{kg} / \mathrm{m}^{2}\right)$ & $22.58 \pm 3.05$ & $23.31 \pm 3.98$ & \\
ASA score & & & \\
I & $8(18.60)$ & $6(13.64)$ & 0.34 \\
II & $30(69.77)$ & $34(77.27)$ & 0.43 \\
III & $5(11.63)$ & $4(9.09)$ & 0.70 \\
History of hypertension & $10(23.26)$ & $9(20.45)$ & 0.75 \\
History of cardiopathy & $5(11.63)$ & $3(5.82)$ & 0.44 \\
History of smoking & $3(6.98)$ & $4(9.09)$ & 0.72 \\
PLOS (days) & $6.56 \pm 1.23$ & $7.02 \pm 2.16$ & 0.23 \\
\hline
\end{tabular}

Values are expressed as the mean \pm standard deviation or $\mathrm{n}(\%) . \mathrm{CV}$, conventional ventilation; PV, protective ventilation; ASA, American Society of Anesthesiology; BMI, body mass index; PLOS, post-operative length of stay.

Table II. Intra-operative data.

\begin{tabular}{|c|c|c|c|}
\hline Parameter & CV group $(n=43)$ & PV group $(n=44)$ & P-value \\
\hline Mechanical ventilation time (min) & $145.97 \pm 60.02$ & $156.15 \pm 50.43$ & 0.41 \\
\hline Operation time (min) & $125.03 \pm 34.23$ & $127.21 \pm 44.26$ & 0.82 \\
\hline Tidal volume (ml) & $535.64 \pm 59.18$ & $440.64 \pm 55.37$ & 0.00 \\
\hline RR (breaths/min) & $12.59 \pm 1.67$ & $13.54 \pm 1.43$ & 0.01 \\
\hline Arousal time (min) & $15.68 \pm 9.34$ & $14.73 \pm 8.69$ & 0.33 \\
\hline Crystalloid volume (ml/kg) & $18.26 \pm 3.41$ & $18.55 \pm 4.17$ & 0.72 \\
\hline Colloid volume $(\mathrm{ml} / \mathrm{kg}$ & $9.29 \pm 2.00$ & $9.14 \pm 1.79$ & 0.71 \\
\hline Urine output (ml/kg) & $1.38 \pm 0.57$ & $1.41 \pm 0.65$ & 0.79 \\
\hline Blood loss (ml) & $83.82 \pm 27.38$ & $89.25 \pm 34.87$ & 0.42 \\
\hline
\end{tabular}

Values are expressed as the mean \pm standard deviation. $\mathrm{CV}$, conventional ventilation; PV, protective ventilation; RR, respiratory rate.

oxygenation function, as well as a reduced incidence of PPCs during the first 7 days following surgery in patients undergoing laparoscopic total hysterectomy. However, during the peri-operative period, no significant differences were observed in terms of PLOS and inflammatory factors between patients managed using the different ventilatory strategies.

Laparoscopic total hysterectomy is widely used and its advantages are generally accepted to include less invasive surgery, better cosmetic results and reduced duration of hospital stay on the basis of the surgical technique in most advanced facilities. Despite this, certain features of laparoscopic techniques, including the Trendelenburg position and induction of pneumoperitoneum with $\mathrm{CO}_{2}$, have been reported to be potential risk factors for an increased incidence of PPC (36).

The beneficial effects of LPV have been demonstrated in patients with healthy lungs during general anesthesia $(10,37)$. LPV has also been indicated to be superior in patients with severe lung injury and ARDS in ICU settings. Application of PEEP, as an important component of LPV, is currently the primary strategy by which to minimize dynamic strain for established ARDS. However, the continued high mortality rate of ARDS indicates that the current PEEP strategies are not always effective (20).

Therefore, the aims of the current study were to incorporate personalized PEEP into laparoscopic total hysterectomy surgery and to evaluate the effects of intra-operative individualized LPV on clinical outcomes compared with those of conventional mechanical ventilation. It was hypothesized that individualized LPV may improve lung oxygenation function compared to conventional mechanical ventilation, and may therefore reduce the incidence of PPCs in patients undergoing laparoscopic total hysterectomy.

In the present study, individualized PEEP values were determined during a Cstat-directed PEEP titration procedure to protect from hyperdistention, and regular ARM was performed using sustained airway pressure by the CPAP method to prevent atelectasis $(22,38,39)$. In the PV group, ARM did not cause any hemodynamic instability or life-threatening events, including a decrease in systolic blood pressure or heart rate. During the course of mechanical ventilation, LPV is preferred to reduce regional end-inspiratory stretch, which also maintains the lung open to improve gas exchange, obtain better pulmonary oxygenation function and pulmonary Cdyn in healthy adult 
Table III. Occurrence of PPCs during the first 7 days after surgery in the two groups.

\begin{tabular}{lccc}
\hline Item & CV group $(\mathrm{n}=43)$ & PV group $(\mathrm{n}=44)$ & P-value \\
\hline Atelectasis & $4(9.3)$ & $0(0)$ & 0.04 \\
Pleural effusion & $2(4.65)$ & $1(2.27)$ & 0.54 \\
Respiratory infection & $5(11.63)$ & $3(6.82)$ & 0.44 \\
Total incidence of PPCs & $11(25.58)$ & $4(9.09)$ & 0.04
\end{tabular}

Values are expressed as n (\%). PPCs, post-operative pulmonary complications; CV, conventional ventilation; PV, protective ventilation.

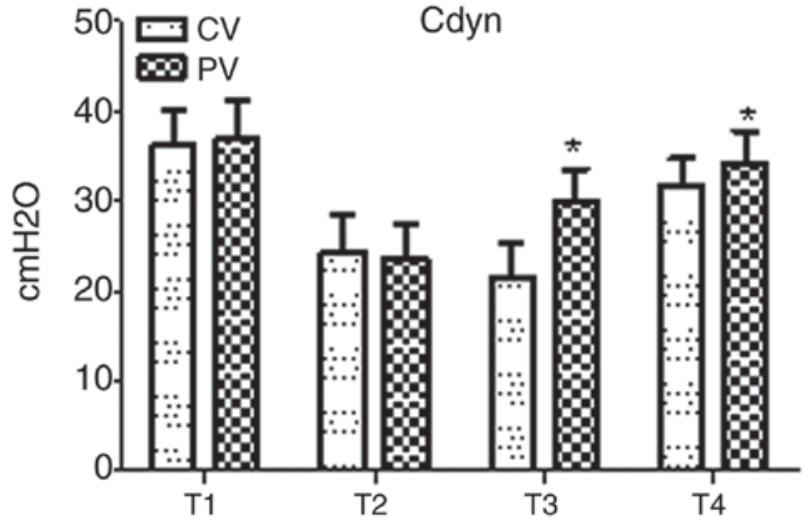

Figure 2. Intra-operative breathing mechanics. In the two groups, Cdyn exhibited a marked but not significant decline at $\mathrm{T} 2$ compared with that at T1. Compared with that in the CV group, Cdyn rose significantly at T3-T4 in the PV group. Values are expressed as the mean \pm standard deviation. ${ }^{*} \mathrm{P}<0.05$ vs. CV group. Time-points: T1, after endotracheal intubation; $\mathrm{T} 2$, 10 min after pneumoperitoneum; T3, 60 min after pneumoperitoneum; T4, $10 \mathrm{~min}$ after pneumoperitoneum was stopped. CV, conventional ventilation; Cdyn, pulmonary dynamic compliance; PV, protective ventilation.

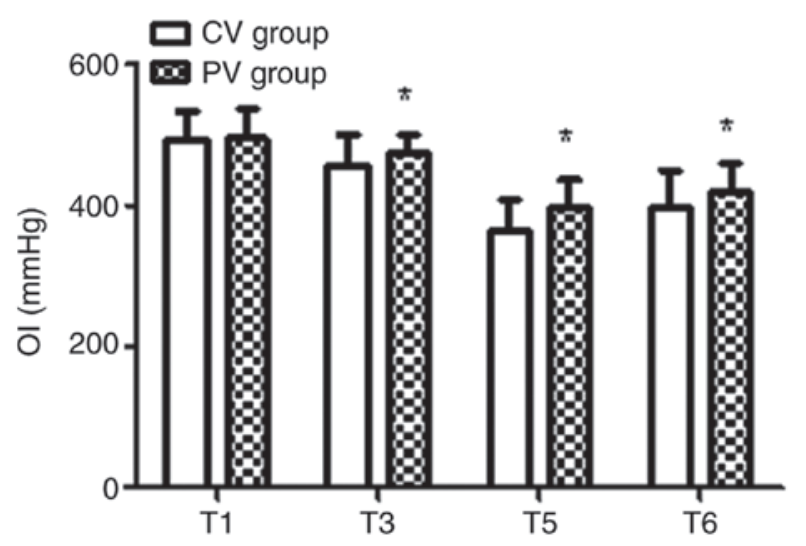

Figure 3. Intra-operative and post-operative pulmonary OI. In the PV group, the OI value was higher compared with that in the CV group at T3, T5 and T6. Values are expressed as the mean \pm standard deviation. ${ }^{*} \mathrm{P}<0.05$ vs. $\mathrm{CV}$ group. Time-points: T1, after endotracheal intubation; T3, 60 min after pneumoperitoneum; T5, 30 min after tracheal extubation; T6, on the day after the surgery. OI, oxygen index; $\mathrm{CV}$, conventional ventilation; $\mathrm{PV}$, protective ventilation.

lungs. The patients in the PV group exhibited improved arterial oxygenation and peripheral oxygen saturation in the peri-operative period, which is in line with the results of a study by Severgnini et al (40), implying that a low TV with adequate PEEP is beneficial for avoiding deoxygenation.

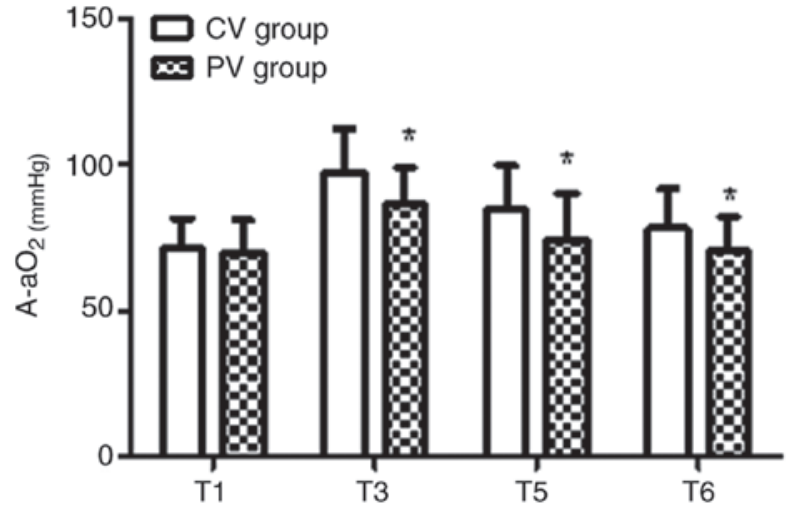

Figure 4. Intra-operative and post-operative pulmonary $\mathrm{A}-\mathrm{aO}_{2}$. In the PV group, the $\mathrm{A}-\mathrm{aO}_{2}$ was lower compared with that in the $\mathrm{CV}$ group at T3, T5 and T6. Values are expressed as the mean \pm standard deviation. ${ }^{*} \mathrm{P}<0.05$ vs. $\mathrm{CV}$ group. Time-points: T1, after endotracheal intubation; T3, $60 \mathrm{~min}$ after pneumoperitoneum; T5, 30 min after tracheal extubation; T6, on the day after the surgery. $\mathrm{A}-\mathrm{aO}_{2}$, alveolar-arterial differences for oxygen; $\mathrm{CV}$, conventional ventilation; PV, protective ventilation.

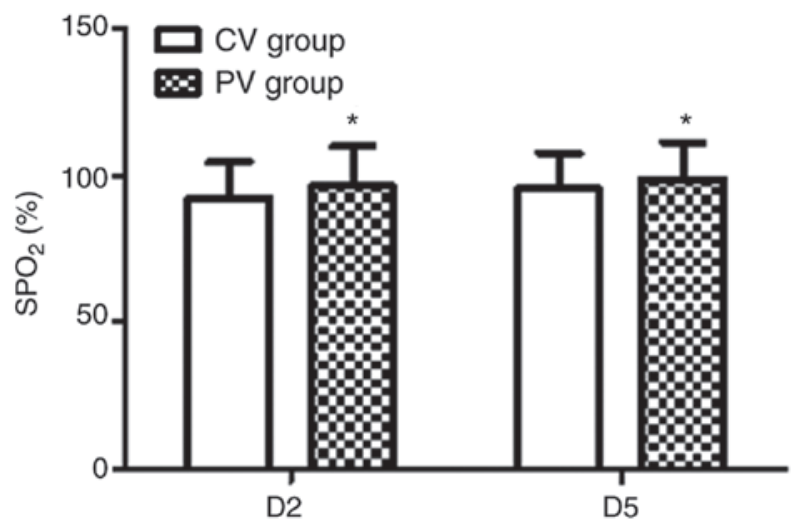

Figure 5. Post-operative $\mathrm{SPO}_{2}$. In the PV group, the $\mathrm{SPO}_{2}$ was higher compared with that in the CV group at D2 and D5. Values are expressed as the mean \pm standard deviation. ${ }^{*} \mathrm{P}<0.05$ vs. $\mathrm{CV}$ group. $\mathrm{CV}$, conventional ventilation; $\mathrm{PV}$, protective ventilation; $\mathrm{SPO}_{2}$, saturation of pulse oxygenation; D2/5, day $2 / 5$ following surgery.

In the present study, individualized LPV strategy was beneficial in the early post-operative period. Certain adverse effects, including atelectasis, pleural effusion and respiratory infection, are linked to mechanical ventilation. Atelectasis and pleural effusion are diagnosed by chest X-ray or CT. In the present study, pneumothorax, bronchospasm, respiratory failure and aspiration pneumonitis were not observed in the 


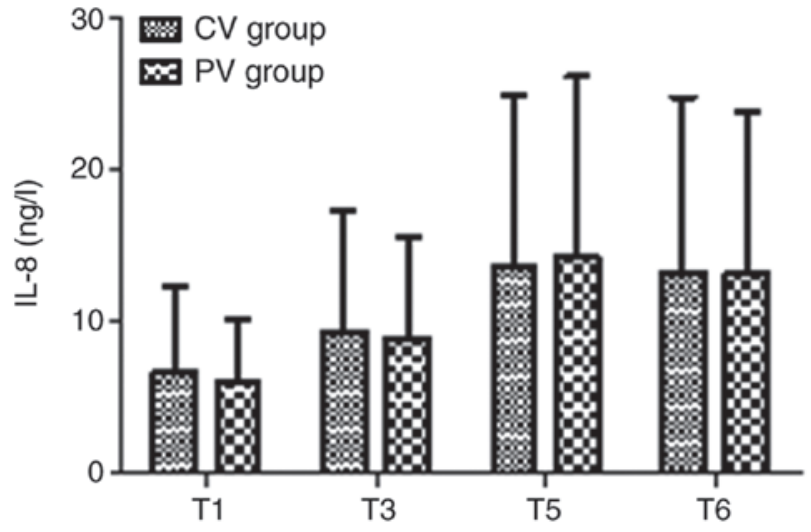

Figure 6. Levels of IL-8 in the two groups. The levels of the inflammatory factor IL-8 were not significantly different between the two groups at any of the time-points. Values are expressed as the mean \pm standard deviation Time-points: T1, after endotracheal intubation; T3, 60 min after pneumoperitoneum; T5, 30 min after tracheal extubation; T6, on the day after the surgery. $\mathrm{CV}$, conventional ventilation; PV, protective ventilation; IL, interleukin.

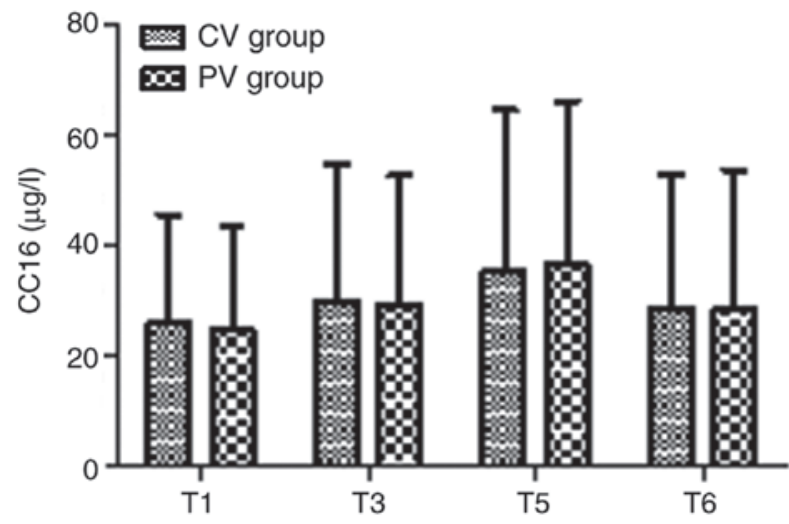

Figure 7. Levels of CC16 in the two groups. The levels of the inflammatory factor CC16 were not significantly different between the two groups at any of the time-points. Values are expressed as the mean \pm standard deviation. T1, after endotracheal intubation; T3,60 min after pneumoperitoneum; T5,30 min after tracheal extubation; T6, on the day after the surgery; CV, conventiona ventilation; PV, protective ventilation; CC16, Clara cell secretory protein 16 .

two groups. Atelectasis, pleural effusion and respiratory infection occurred in the two groups; however, no significant difference was present in the incidence of pleural effusion and respiratory infection. The rate of post-operative atelectasis was higher in the $\mathrm{CV}$ group compared with that in the PV group (9.3 vs. $0 \%$; $\mathrm{P}<0.05)$, as evaluated using chest $\mathrm{X}$-ray or CT, and the total incidence rate of PPCs during the early period after the operation in the $\mathrm{CV}$ group was also higher compared with that in the PV group $(25.58$ vs. $9.09 \%$; $\mathrm{P}<0.05)$. These results suggest that LPV with individualized PEEP and regular RM during surgery may contribute to the prevention of ventilation-induced atelectasis and to improve pulmonary oxygenation function.

The results of the present study are consistent with those of previous studies (41). However, as the sample size was small, it was not possible to demonstrate a notable decrease in the incidence of long-term major PPCs.

Although LPV was indicated to be associated with reduced PPCs in the present study, no significant difference was obtained in the serum levels of the inflammatory factors IL-8 and CC16 during and after surgery, and the PLOS was similar between the groups. Previous studies demonstrated that invasive mechanical ventilation may change the course of existing inflammation in patients with ARDS. The results of the present study suggest that the difference in ventilatory strategy during surgery alone does not change the inflammatory process in patients without a history of pulmonary disease. It is possible that the ventilation period may have been too short to produce changes in certain inflammatory mediators due to the long duration of transcriptional and translational processes. In the present study, the same intra-operative infusion was adopted in the two groups to exclude any differences in inflammatory markers. Even though intra-operative infusion may have affected the serum levels of IL-8 and CC16, the influence was identical in the two groups.

Laparoscopic total hysterectomy is considered to be a minimally invasive surgical procedure and the increased inflammatory biomarkers in the two groups in the post-operative period (T3, T5 and T6) may have been due to the surgery itself. Ventilatory strategy alterations during surgery alone are not considered to be sufficient to change the inflammatory processes in patients without a history of pulmonary disease. All patients received routine physiotherapy and antibiotics according to the standard care at our hospital at the post-operative stage, including encouragement to cough, pats on the back and turning over. The inflammatory factors increased slightly, and there was no significant difference between the two groups. The results of the present study are similar to those of previous studies with regard to inflammatory mediators $(42,43)$ and PLOS $(44)$.

The present study had certain potential limitations. First, the effects of ventilation strategies on major PPCs are not a major focus of the present study, as the sample size was too small. Furthermore, the study included only female patients, as a gynecological disease was being treated. In addition, the patients were only followed up for the first 7 days after surgery. Hence, longitudinal studies are required to demonstrate the long-term clinical impact on the incidence rates of PPCs.

In conclusion, the results of the present study demonstrated that protective ventilation strategies in laparoscopic total hysterectomy with low $\mathrm{TV}$, intra-operative individualized PEEP and regular ARM during surgery are able to significantly improve pulmonary oxygenation function and reduce the total incidence of PPCs and atelectasis. However, larger prospective randomized trials on different types of surgery, including mixed-gender populations and long-term evaluation after surgery, are recommended in the future to determine the benefit of low TV together with individualized optimal PEEP for the patient.

\section{Acknowledgements}

Not applicable.

\section{Funding}

The present study was supported by the Public Welfare Technology Research Project of the Huzhou Science and Technology Bureau (grant no. 2019GYB55) and the Zhejiang 
Province Medical Science and Technology Plan (grant no. 2020KY932).

\section{Availability of data and materials}

The datasets used and/or analyzed during the current study are available from the corresponding author on reasonable request.

\section{Authors' contributions}

JL contributed to the study conception and design, data acquisition, statistical analysis, data interpretation and drafting of the manuscript. XH contributed to the statistical analysis and data interpretation and revised the manuscript. SH contributed to the study design and data acquisition and revised the manuscript. ZM contributed to the study conception and design, performed data interpretation and revised the manuscript. $\mathrm{HH}$ contributed to data acquisition and revised the manuscript. All authors read and approved the final manuscript.

\section{Ethics approval and consent to participate}

This study was approved by the Institutional Ethics Committee of Huzhou Maternal and Child Healthcare Hospital (Huzhou, China) and was performed in accordance with the ethical standards set by the 1964 Declaration of Helsinki and its later amendments. Written informed consent was obtained from all patients or their legal representative prior to enrollment in the study.

\section{Patient consent for publication}

Not applicable.

\section{Competing interests}

The authors declare that they have no competing interests.

\section{References}

1. Slutsky AS and Ranieri VM: Ventilator-induced lung injury. N Engl J Med 369: 2126-2136, 2013.

2. Ricard JD, Dreyfuss D and Saumon G: Ventilator-induced lung injury. Eur Respir J Suppl 42: 2s-9s, 2003.

3. Ware LB, Koyama T, Zhao Z, Janz DR, Wickersham N, Bernard GR, May AK, Calfee CS and Matthay MA: Biomarkers of lung epithelial injury and inflammation distinguish severe sepsis patients with acute respiratory distress syndrome. Crit Care 17: R253, 2013.

4. Famous KR, Delucchi K, Ware LB, Kangelaris KN, Liu KD, Thompson BT and Calfee CS; ARDS Network: Acute respiratory distress syndrome subphenotypes respond differently to randomized fluid management strategy. Am J Respir Crit Care Med 195: 331-338, 2017.

5. Bos LD, Schouten LR, van Vught LA, Wiewel MA, Ong DSY, Cremer O, Artigas A, Martin-Loeches I, Hoogendijk AJ, van der Poll T, et al: Identification and validation of distinct biological phenotypes in patients with acute respiratory distress syndrome by cluster analysis. Thorax 72: 876-883, 2017.

6. Broeckaert F and Bernard A: Clara cell secretory protein (CC16): Characteristics and perspectives as lung peripheral biomarker. Clin Exp Allergy 30: 469-475, 2000.

7. Fernandez-Bustamante A, Klawitter J, Repine JE, Agazio A, Janocha AJ,ShahC,Moss M,Douglas IS,TranZV,Erzurum SC, et al: Early effect of tidal volume on lung injury biomarkers in surgical patients with healthy lungs. Anesthesiology 121: 469-481, 2014
8. Wutzler S, Lehnea T, Laurer H, Lehnert M, Becker M, Henrich D, Vogl T and Marzi I: Circulating levels of clara cell protein 16 but not surfactant protein D identify and quantify lung damage in patients with multiple injuries. J Tranma 71: E31-E36, 2011.

9. Futier E, Constantin JM, Paugam-Burtz C, Pascal J, Eurin M, Neuschwander A, Marret E, Beaussier M, Gutton C, Lefrant JY, et al: A trial of intraoperative low-tidal-volume ventilation in abdominal surgery. N Engl J Med 369: 428-437, 2013.

10. PROVE Network Investigators for the Clinical Trial Network of the European Society of Anaesthesiology, Hemmes SN, Gama de Abreu M, Pelosi P and Schultz MJ: High versus low positive end-expiratory pressure during general anaesthesia for open abdominal surgery (PROVHILO trial): A multicentre randomised controlled trial. Lancet 384: 495-503, 2014.

11. Brower RG, Lanken PN, MacIntyre N, Matthay MA, Morris A, Ancukiewicz M, Schoenfeld D and Thompson BT; National Heart, Lung, and Blood Institute ARDS Clinical Trials Network: Higher versus lower positive end-expiratory pressures in patients with the acute respiratory distress syndrome. N Engl J Med 351: 327-336, 2004.

12. Jaber S, Coisel Y, Chanques G, Futier E, Constantin J, Michelet P, Beaussier M, Lefrant JY, Allaouchiche B, Capdevila X and Marret E: A multicentre observational study of intra-operative ventilatory management during general anaesthesia: Tidal volumes and relation to body weight. Anaesthesia 67: 999-1008, 2012.

13. Haliloglu M, Bilgili B, Ozdemir M, Umuroglu T and Bakan N: Low tidal volume positive end-expiratory pressure versus high tidal volume zero-positive end-expiratory pressure and postoperative pulmonary functions in robot-assisted laparoscopic radical prostatectomy. Med Princ Pract 26: 573-578, 2017.

14. Hansen JK, Anthony DG, Li L, Wheeler D, Sessler DI and Bashour CA: Comparison of positive end-expiratory pressure of 8 versus $5 \mathrm{~cm} \mathrm{H}_{2} \mathrm{O}$ on outcome after cardiac operations. J Intensive Care Med 6: 338-343, 2015.

15. Reis Miranda D, Gommers D, Struijs A, Dekker R, Mekel J, Feelders R, Lachmann B and Bogers AJ: Ventilation according to the open lung concept attenuates pulmonary inflammatory response in cardiac surgery. Eur J Cardiothorac Surg 28: 889-895, 2005.

16. García-Delgado M, Navarrete-Sánchez I and Colmenero M: Preventing and managing perioperative pulmonary complications following cardiac surgery. Curr Opin Anaesthesiol 27: 146-152, 2014.

17. Serpa Neto A, Hemmes SN, Barbas CS, Beiderlinden M, Biehl M, Binnekade JM, Canet J, Fernandez-Bustamante A, Futier E, Gajic O, et al: Protective versus conventional ventilation for surgery. A systematic review and individual patient data meta-analysis. Anesthesiology 123: 66-78, 2015.

18. Zhang Z, Hu X, Zhang X, Zhu X, Chen L, Zhu L, Hu C and Du B; China Critical Care Clinical Trials Group (CCCCTG): Lung protective ventilation in patients undergoing major surgery: A systematic review incorporating a Bayesian approach. BMJ Open 5: e007473, 2015.

19. Yang D, Grant MC, Stone A, Wu CL and Wick EC: A meta-analysis of intraoperative ventilation strategies to prevent pulmonary complications: Is tidal volume alone sufficient to protect healthy lungs? Ann Surg 263: 881-887, 2016.

20. Villar J, Blanco J and Kacmarek RM: Current incidence and outcome of the acute respiratory distress syndrome. Curr Opin Crit Care 22: 1-6, 2016.

21. Levin MA, McCormick PJ, Lin HM, Hosseinian L and Fischer GW: Low intraoperative tidal volume ventilation with minimal PEEP is associated with increased mortality. Br J Anaesth 113: 97-108, 2014.

22. Vargas M, Sutherasan Y, Gregoretti C and Pelosi P: PEEP role in ICU and operating room: From pathophysiology to clinical practice. ScientificWorldJournal 2014: 852356, 2014.

23. Menendez C, Martinez-Caro L, Moreno L, Nin N, Moral-Sanz J, Morales D, Cogolludo A, Esteban A, Lorente JA and Perez-Vizcaino F: Pulmonary vascular dysfunction induced by high tidal volume mechanical ventilation. Crit Care Med 41: e149-e155, 2013.

24. Nieman GF, Satalin J, Andrews P, Aiash H, Habashi NM and Gatto LA: Personalizing mechanical ventilation according to physiologic parameters to stabilize alveoli and minimize ventilator induced lung injury (VILI). Intensive Care Med Exp 5: 8, 2017.

25. Kishida M, Yamada Y, Inayama E, Kitamura M, Nishino T, Ota K, Shintani A and Ikenoue T: Effectiveness of music therapy for alleviating pain during haemodialysis access cannulation for patients undergoing haemodialysis: A multi-facility, single-blind, randomised controlled trial. Trials 20: 631, 2019. 
26. Lawrence VA, Cornell JE and Smetana GW; American College of Physicians: Strategies to reduce postoperative pulmonary complications after noncardiothoracic surgery: Systematic review for the American college of physicians. Ann Intern Med 144: 596-608, 2006.

27. Güldner A, Kiss T, Serpa Neto A, Hemmes SN, Canet J, Spieth PM, Rocco PR, Schultz MJ, Pelosi P and Gama de Abreu M: Intraoperative protective mechanical ventilation for prevention of postoperative pulmonary complications: A comprehensive review of the role of tidal volume, positive end-expiratory pressure, and lung recruitment maneuvers. Anesthesiology 123: 692-713, 2015.

28. Ruszkai Z, Kiss E, László I, Gyura F, Surány E, Bartha PT, Bokrétás GP, Rácz E, Buzogány I, Bajory Z, et al: Effects of intraoperative PEEP optimization on postoperative pulmonary complications and the inflammatory response: Study protocol for a randomized controlled trial. Trials 18: 375, 2017.

29. Marret E, Cinotti R, Berard L, Piriou V, Jobard J, Barrucand B, Radu D, Jaber S and Bonnet F; and the PPV study group: Protective ventilation during anaesthesia reduces major postoperative complications after lung cancer surgery: A double-blind randomised controlled trial. Eur J Anaesthesiol 35: 727-735, 2018.

30. Okada S, Ito K, Shimada J, Kato D, Shimomura M, Tsunezuka H, Miyata N, Ishihara S, Furuya T and Inoue M: Clinical application of postoperative non-invasive positive pressure ventilation after lung cancer surgery. Gen Thorac Cardiovasc Surg 66: 66: 565-572, 2018

31. Jammer I, Wickboldt N, Sander M, Smith A, Schultz MJ, Pelosi P, Leva B, Rhodes A, Hoeft A, Walder B, et al: Standards for definitions and use of outcome measures for clinical effectiveness research in perioperative medicine: European perioperative clinical outcome (EPCO) definitions: A statement from the ESA-ESICM joint taskforce on perioperative outcome measures. Eur J Anaesthesiol 32: 88-105, 2015.

32. Gallart L and Canet J: Post-operative pulmonary complications: Understanding definitions and risk assessment. Best Pract Res Clin Anaesthesiol 29: 315-330, 2015.

33. Arslantas MK, Kara HV, Tuncer BB, Yildizeli B, Yuksel M, Bostanci K, Bekiroglu N, Kararmaz A, Cinel I and Batirel HF: Effect of the amount of intraoperative fluid administration on postoperative pulmonary complications following anatomic lung resections. J Thorac Cardiovasc Surg 149: 314-320, 321.e1, 2015.

34. Mommers EHH, Wegdam JA, van der Wolk S, Nienhuijs SW and de Vries Reilingh TS: Impact of hernia volume on pulmonary complications following complex hernia repair. J Surg Res 211 8-13, 2017.
35. Faul F, Erdfelder E, Buchner A and Lang AG: Statistical power analyses using $G^{*}$ Power 3.1: Tests for correlation and regression analyses. Behav Res Methods 41: 1149-1160, 2009.

36. Smetana GW, Lawrence VA and Cornell JE; American College of Physicians: Preoperative pulmonary risk stratification for noncardiothoracic surgery: Systematic review for the American college of physicians. Ann Intern Med 144: 581-595, 2006.

37. Futier $\mathrm{E}$ and Jaber S: Lung-protective ventilation in abdominal surgery. Curr Opin Crit Care 20: 426-430, 2014.

38. Sutherasan Y, Vargas M and Pelosi P: Protective mechanical ventilation in the non-injured lung: Review and meta-analysis. Crit Care 18: 211, 2014.

39. Pelosi P, Gama de Abreu M and Rocco PR: New and conventional strategies for lung recruitment in acute respiratory distress syndrome. Crit Care 14: 210, 2010.

40. Severgnini P, Selmo G, Lanza C, Chiesa A, Frigerio A, Bacuzzi A, Dionigi G, Novario R, Gregoretti C, de Abreu MG, et al: Protective mechanical ventilation during general anesthesia for open abdominal surgery improves postoperative pulmonary function. Anesthesiology 118: 1307-1321, 2013

41. Serpa Neto A, Cardoso SO, Manetta JA, Pereira VG, Espósito DC, Pasqualucci Mde O, Damasceno MC and Schultz MJ: Association between use of lung-protective ventilation with lower tidal volumes and clinical outcomes among patients without acute respiratory distress syndrome: A meta-analysis. JAMA 308: 1651-1659, 2012

42. Lee JH, Bae JI, Jang YE, Kim EH, Kim HS and Kim JT: Lung protective ventilation during pulmonary resection in children: A prospective, single-centre, randomised controlled trial. Br J Anaesth 122: 692-701, 2019.

43. Kokulu S, Günay E, Baki ED, Ulasli SS, Yilmazer M, Koca B, Arıöz DT, Ela Y and Sivaci RG: Impact of a lung-protective ventilatory strategy on systemic and pulmonary inflammatory responses during laparoscopic surgery: Is it really helpful? Inflammation 38: 361-367, 2014.

44. Memtsoudis SG, Bombardieri AM, Ma Y and Girardi FP: The effect of low versus high tidal volume ventilation on inflammatory markers in healthy individuals undergoing posterior spine fusion in the prone position: A randomized controlled trial. J Clin Anesth 24: 263-269, 2012

This work is licensed under a Creative Commons Attribution-NonCommercial-NoDerivatives 4.0 International (CC BY-NC-ND 4.0) License. 